\title{
Adjuvant Ovarian Suppression in Premenopausal Breast Cancer
}

\author{
Prudence A. Francis, M.D., Meredith M. Regan, Sc.D., Gini F. Fleming, M.D., \\ István Láng, M.D., Eva Ciruelos, M.D., Meritxell Bellet, M.D., Hervé R. Bonnefoi, M.D., \\ Miguel A. Climent, M.D., Gian Antonio Da Prada, M.D., Harold J. Burstein, M.D., Ph.D., \\ Silvana Martino, D.O., Nancy E. Davidson, M.D., Charles E. Geyer, Jr., M.D., \\ Barbara A. Walley, M.D., Robert Coleman, M.B., B.S., M.D., Pierre Kerbrat, M.D., \\ Stefan Buchholz, M.D., James N. Ingle, M.D., Eric P. Winer, M.D., \\ Manuela Rabaglio-Poretti, M.D., Rudolf Maibach, Ph.D., Barbara Ruepp, Pharm.D., \\ Anita Giobbie-Hurder, M.S., Karen N. Price, B.S., Marco Colleoni, M.D., \\ Giuseppe Viale, M.D., Alan S. Coates, M.D., Aron Goldhirsch, M.D., \\ and Richard D. Gelber, Ph.D., for the SOFT Investigators \\ and the International Breast Cancer Study Group*
}

A BSTRACT

The authors' affiliations are listed in the Appendix. Address reprint requests to Dr. Francis at the Peter MacCallum Cancer Centre, Locked Bag 1, A'Beckett St., Melbourne, VIC 8006, Australia, or at ibcsgcc@ibcsg.org.

Drs. Francis and Regan contributed equally to this article.

*A complete list of investigators in the Suppression of Ovarian Function Trial (SOFT) and the International Breast Cancer Study Group is provided in the Supplementary Appendix, available at NEJM.org.

This article was published on December 11 2014, at NEJM.org.

N Engl J Med 2015;372:436-46. DOI: 10.1056/NEJMoal412379 Copyright (c) 2014 Massachusetts Medical Society.

\section{BACKGROUND}

Suppression of ovarian estrogen production reduces the recurrence of hormonereceptor-positive early breast cancer in premenopausal women, but its value when added to tamoxifen is uncertain.

METHODS

We randomly assigned 3066 premenopausal women, stratified according to prior receipt or nonreceipt of chemotherapy, to receive 5 years of tamoxifen, tamoxifen plus ovarian suppression, or exemestane plus ovarian suppression. The primary analysis tested the hypothesis that tamoxifen plus ovarian suppression would improve disease-free survival, as compared with tamoxifen alone. In the primary analysis, $46.7 \%$ of the patients had not received chemotherapy previously, and $53.3 \%$ had received chemotherapy and remained premenopausal.

\section{RESULTS}

After a median follow-up of 67 months, the estimated disease-free survival rate at 5 years was $86.6 \%$ in the tamoxifen-ovarian suppression group and $84.7 \%$ in the tamoxifen group (hazard ratio for disease recurrence, second invasive cancer, or death, 0.83 ; $95 \%$ confidence interval $[\mathrm{CI}], 0.66$ to $1.04 ; \mathrm{P}=0.10$ ). Multivariable allowance for prognostic factors suggested a greater treatment effect with tamoxifen plus ovarian suppression than with tamoxifen alone (hazard ratio, $0.78 ; 95 \% \mathrm{CI}, 0.62$ to 0.98). Most recurrences occurred in patients who had received prior chemotherapy, among whom the rate of freedom from breast cancer at 5 years was $82.5 \%$ in the tamoxifen-ovarian suppression group and $78.0 \%$ in the tamoxifen group (hazard ratio for recurrence, $0.78 ; 95 \%$ CI, 0.60 to 1.02). At 5 years, the rate of freedom from breast cancer was $85.7 \%$ in the exemestane-ovarian suppression group (hazard ratio for recurrence vs. tamoxifen, 0.65 ; $95 \%$ CI, 0.49 to 0.87 ).

CONCLUSIONS

Adding ovarian suppression to tamoxifen did not provide a significant benefit in the overall study population. However, for women who were at sufficient risk for recurrence to warrant adjuvant chemotherapy and who remained premenopausal, the addition of ovarian suppression improved disease outcomes. Further improvement was seen with the use of exemestane plus ovarian suppression. (Funded by Pfizer and others; SOFT ClinicalTrials.gov number, NCT00066690.) 
DJUVANT ENDOCRINE THERAPY WITH tamoxifen has been recommended for premenopausal women with hormonereceptor-positive breast cancer (positive for estrogen receptor, progesterone receptor, or both) during the past 15 years. ${ }^{1,2}$ The value of therapeutic suppression of ovarian estrogen production in premenopausal women who receive tamoxifen is uncertain. ${ }^{3}$ The American Society of Clinical Oncology endorsed guidelines recommending that ovarian ablation or suppression (hereafter, ovarian suppression) not be added routinely to adjuvant therapy in premenopausal women. ${ }^{4}$ Chemotherapy-induced ovarian suppression (amenorrhea) is correlated with a reduced risk of relapse $\mathrm{e}^{5-7}$ but is less likely to be achieved in very young women. International consensus guidelines for breastcancer management in young women suggested that the addition of a gonadotropin-releasing hormone (GnRH) agonist to tamoxifen be discussed on an individualized basis. ${ }^{8}$

In 2003, the International Breast Cancer Study Group (IBCSG) initiated two randomized, phase 3 trials, the Suppression of Ovarian Function Trial (SOFT) and the Tamoxifen and Exemestane Trial (TEXT), involving premenopausal women with hormone-receptor-positive early breast cancer. SOFT was designed to determine the value of adding ovarian suppression to tamoxifen and to determine the role of adjuvant therapy with the aromatase inhibitor exemestane plus ovarian suppression in premenopausal women. Here we report the results of the planned primary analysis in SOFT $^{9}$ comparing adjuvant tamoxifen plus ovarian suppression with tamoxifen alone after a median follow-up of 67 months.

\section{METHODS}

\section{PATIENTS}

The trial was designed to evaluate adjuvant endocrine therapy in women who remained premenopausal after the completion of adjuvant or neoadjuvant chemotherapy and in premenopausal women for whom adjuvant tamoxifen alone was considered suitable treatment. Eligibility criteria included documented premenopausal status, operable breast cancer, and tumor that expressed estrogen or progesterone receptors in at least $10 \%$ of the cells (see the Supplementary Appendix, available with the full text of this article at NEJM.org).

Patients had to have undergone either a total mastectomy with subsequent optional radiother- apy or breast-conserving surgery with subsequent radiotherapy. Either axillary dissection or a sentinel-node biopsy was required. Patients who had not received chemotherapy underwent randomization within 12 weeks after definitive surgery. Patients who received chemotherapy before randomization and remained premenopausal were enrolled within 8 months after completing chemotherapy, once a premenopausal estradiol level was confirmed by a local laboratory. Patients were allowed to receive adjuvant oral endocrine therapy before randomization.

\section{STUDY DESIGN}

Women were randomly assigned to receive oral tamoxifen at a dose of $20 \mathrm{mg}$ daily, tamoxifen plus ovarian suppression, or oral exemestane (Aromasin, Pfizer) at a dose of $25 \mathrm{mg}$ daily plus ovarian suppression. Treatment was for 5 years from the date of randomization, according to the study protocol, available at NEJM.org. Ovarian suppression was achieved by choice of triptorelin (Decapeptyl Depot [triptorelin acetate], Ipsen; or Trelstar Depot [triptorelin pamoate], Debio) at a dose of $3.75 \mathrm{mg}$ administered by means of intramuscular injection every 28 days, bilateral oophorectomy, or bilateral ovarian irradiation. Patients receiving triptorelin could subsequently opt to undergo oophorectomy or irradiation. Randomization was performed by means of the IBCSG Internet-based system and was stratified according to prior chemotherapy (yes vs. no), lymphnode status (positive vs. negative), and intended initial method of ovarian suppression, if assigned. The assessments of the patients and the recording of adverse events followed a regular schedule (see the Supplementary Appendix).

The primary end point was disease-free survival, defined as the time from randomization to the first appearance of one of the following: recurrence of invasive breast cancer (local, regional, or distant), invasive contralateral breast cancer, second (nonbreast) invasive cancer, or death without recurrence or second cancer. Secondary end points included the interval without breast cancer, defined as the time from randomization to the recurrence of invasive breast cancer (local, regional, or distant) or invasive contralateral breast cancer; the interval from randomization to the recurrence of breast cancer at a distant site; and overall survival, defined as the time from randomization to death from any cause.

The ethics committee at each participating 
center approved the study protocol, and all the patients provided written informed consent. The IBCSG was responsible for the trial design, data collection, and analysis. Pfizer and Ipsen, the respective manufacturers of exemestane and triptorelin, donated the study drugs; neither manufacturer imposed restrictions with respect to the trial data. The manuscript was written solely by the authors, who vouch for the data and analyses reported and for the fidelity of the study to the protocol. The steering committee (which included employees of Pfizer and Ipsen) reviewed the manuscript and made the decision to submit it for publication.

\section{STATISTICAL ANALYSIS}

The original statistical analysis plan for SOFT was to assess disease-free survival between the treatment groups with three pairwise comparisons. ${ }^{9}$ The design assumed the enrollment of predominantly very young women who remained premenopausal after chemotherapy and would have an expected disease-free survival rate at 5 years of $67 \%$ when treated with tamoxifen, on the basis of outcomes for patients younger than 35 years of age in previous trials. ${ }^{9}$ The enrolled patients were older and had lower-risk characteristics than anticipated, and the rate of disease-free survival was higher than expected. A protocol amendment to the analysis plan was adopted in 2011, designating the test of the superiority of tamoxifen plus ovarian suppression over tamoxifen alone as the primary analysis for SOFT. ${ }^{9}$ We calculated that with an estimated 186 events of disease recurrence, second invasive cancer, or death in the two treatment groups after a median follow-up of 5 years, the study would have at least $80 \%, 69 \%$, and $52 \%$ power to detect reductions in risk of $33.5 \%, 30 \%$, and $25 \%$, respectively, with tamoxifen plus ovarian suppression versus tamoxifen alone, at a two-sided alpha level of 0.05 . The comparison of exemestane plus ovarian suppression with tamoxifen alone became a secondary objective, and the comparison of exemestane plus ovarian suppression with tamoxifen plus ovarian suppression was analyzed by means of a combined analysis with the TEXT data. ${ }^{10}$

Analyses were performed according to the

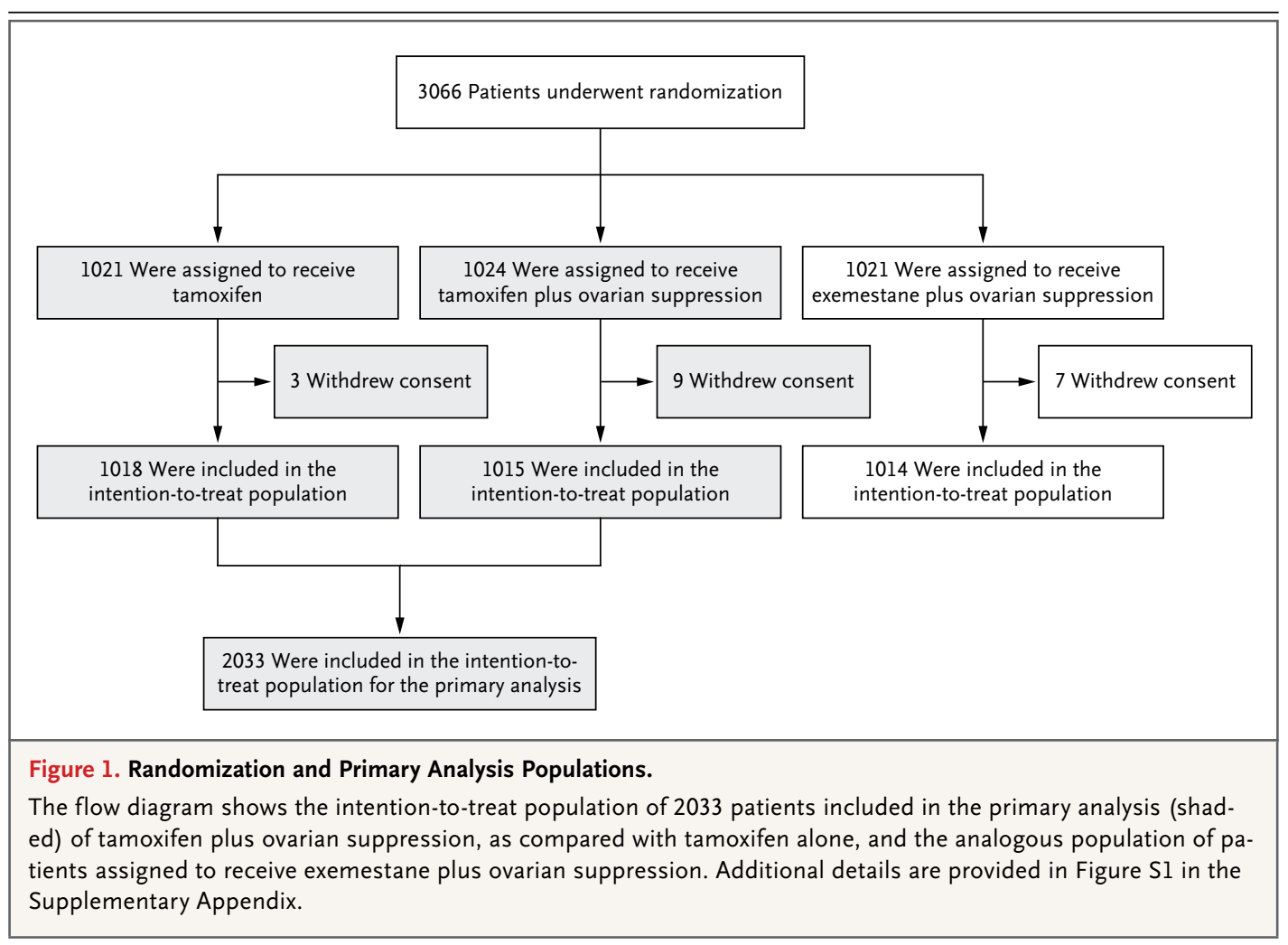


intention-to-treat principle. Kaplan-Meier estimates of time-to-event end points were calculated. Hypothesis tests compared the two groups with the use of log-rank tests, stratified according to prior use of chemotherapy (yes vs. no) and lymph-node status (positive vs. negative). Stratified Cox proportional-hazards regression was used to estimate hazard ratios and $95 \%$ confidence intervals. In prespecified secondary analyses, heterogeneity of the treatment effect according to subgroup was investigated by tests of treatment-by-covariate interaction, and an adjusted hazard ratio for the treatment effect was estimated.

\section{RESULTS}

\section{STUDY POPULATION}

From December 2003 through January 2011, we randomly assigned 1021 premenopausal women to tamoxifen, 1024 to tamoxifen plus ovarian suppression, and 1021 to exemestane plus ovarian suppression. After exclusions, 2033 women were included in the intention-to-treat population for the primary analysis comparing tamoxifen plus ovarian suppression with tamoxifen alone (Fig. 1, and Fig. S1 in the Supplementary Appendix). The median age of the patients was 43 years (Table 1). A total of $46.7 \%$ of the pa-

\begin{tabular}{|c|c|c|c|}
\hline Characteristic & $\begin{array}{l}\text { No Chemotherapy } \\
\qquad(\mathrm{N}=949)\end{array}$ & $\begin{array}{l}\text { Prior Chemotherapy } \\
\qquad(\mathrm{N}=1084)\end{array}$ & $\begin{array}{l}\text { Overall } \\
(N=2033)\end{array}$ \\
\hline \multicolumn{4}{|l|}{ Age at randomization } \\
\hline Median $-y r$ & 46 & 40 & 43 \\
\hline \multicolumn{4}{|l|}{ Distribution - no. (\%) } \\
\hline$<35 \mathrm{yr}$ & $14(1.5)$ & $219(20.2)$ & $233(11.5)$ \\
\hline $35-39 \mathrm{yr}$ & $78(8.2)$ & $309(28.5)$ & $387(19.0)$ \\
\hline $40-49 \mathrm{yr}$ & $702(74.0)$ & $522(48.2)$ & $1224(60.2)$ \\
\hline$\geq 50 \mathrm{yr}$ & $155(16.3)$ & $34(3.1)$ & $189(9.3)$ \\
\hline \multicolumn{4}{|l|}{ Lymph-node status — no. (\%) } \\
\hline Negative & $861(90.7)$ & $463(42.7)$ & $1324(65.1)$ \\
\hline Positive & $88(9.3)$ & $621(57.3)$ & 709 (34.9) \\
\hline \multicolumn{4}{|l|}{ Tumor size - no. (\%)† } \\
\hline$\leq 2 \mathrm{~cm}$ & $806(84.9)$ & $526(48.5)$ & $1332(65.5)$ \\
\hline$>2 \mathrm{~cm}$ & $136(14.3)$ & $513(47.3)$ & 649 (31.9) \\
\hline \multicolumn{4}{|l|}{ Tumor grade - no. $(\%) \ddagger$} \\
\hline 1 & $389(41.0)$ & $151(13.9)$ & $540(26.6)$ \\
\hline 2 & $483(50.9)$ & $523(48.2)$ & $1006(49.5)$ \\
\hline 3 & $65(6.8)$ & $374(34.5)$ & $439(21.6)$ \\
\hline HER2-positive - no. (\%) & $40(4.2)$ & $196(18.1)$ & $236(11.6)$ \\
\hline \multicolumn{4}{|l|}{ Interval from surgery to randomization - mo } \\
\hline Median & 1.8 & 8.0 & 3.2 \\
\hline Interquartile range & $1.2-2.4$ & $5.8-10.3$ & $1.7-8.33$ \\
\hline Endocrine therapy before randomization - no. (\%)』 & $47(5.0)$ & $475(43.8)$ & $522(25.7)$ \\
\hline
\end{tabular}

* A more complete summary is provided in Table S1 in the Supplementary Appendix. Characteristics were well balanced according to treatment assignment (Table S2 in the Supplementary Appendix). The statistical analysis plan did not specify hypothesis testing regarding comparisons between these groups. HER2 denotes human epidermal growth factor receptor 2 . $\uparrow$ Data were missing for 7 patients who did not receive chemotherapy and for 45 who had received chemotherapy previously. Data were missing for 12 patients who did not receive chemotherapy and for 36 who had received chemotherapy previously. $\int$ Oral endocrine therapy before randomization was allowed while premenopausal status was established or reestablished. 
tients had not received chemotherapy previously, and $53.3 \%$ received chemotherapy before randomization and remained premenopausal. Nodepositive disease was present in $34.9 \%$ of the patients.

\section{EFFICACY}

At a median follow-up of 67 months, 299 patients $(14.7 \%)$ had recurrent disease or a second invasive cancer or had died. The rate of diseasefree survival at 5 years was $86.6 \%$ (95\% confidence interval [CI], 84.2 to 88.7) among patients assigned to receive tamoxifen plus ovarian suppression, as compared with $84.7 \%$ (95\% CI, 82.2 to 86.9) among those assigned to tamoxifen alone (hazard ratio for recurrence, second invasive cancer, or death, 0.83 ; 95\% CI, 0.66 to 1.04; $\mathrm{P}=0.10$ ) (Fig. 2A). A total of $58.2 \%$ of the first events involved distant sites, and $12.0 \%$ were second (nonbreast) malignant conditions (Table S3 in the Supplementary Appendix). Planned subgroup analyses did not reveal heterogeneity of treatment effect across most subgroups (Fig. S2 in the Supplementary Appendix). However, the subgroup of patients with human epidermal growth factor receptor 2-positive tumors appeared to have a greater benefit with tamoxifen plus ovarian suppression than with tamoxifen alone. In the multivariable Cox proportional-hazards model, with adjustment for covariates, tamoxifen plus ovarian suppression significantly reduced the hazard of recurrence, a second invasive cancer, or death, as compared with tamoxifen alone (hazard ratio, $0.78 ; 95 \% \mathrm{CI}, 0.62$ to $0.98 ; \mathrm{P}=0.03$ ) (Table $\mathrm{S} 4$ in the Supplementary Appendix).

At 5 years, $88.4 \%$ (95\% CI, 86.1 to 90.3 ) of the patients assigned to receive tamoxifen plus ovarian suppression remained free from breast cancer, as compared with $86.4 \%$ ( $95 \%$ CI, 84.0 to 88.5) of those assigned to receive tamoxifen alone (hazard ratio for recurrence, $0.81 ; 95 \% \mathrm{CI}$, 0.63 to 1.03; $\mathrm{P}=0.09$ ) (Fig. 3A). After adjustment for covariates in the multivariable Cox proportional-hazards model, tamoxifen plus ovarian suppression reduced the hazard of breast-cancer recurrence, as compared with tamoxifen alone (hazard ratio, 0.75 ; $95 \% \mathrm{CI}, 0.59$ to $0.96 ; \mathrm{P}=0.02$ ). Among patients assigned to receive exemestane plus ovarian suppression, $90.9 \%$ (95\% CI, 88.9 to 92.6) remained free from breast cancer at 5 years.
Figure 2 (facing page). Primary Analysis Comparisons of Tamoxifen plus Ovarian Suppression (OS) with Tamoxifen Alone.

Panel A shows Kaplan-Meier estimates of disease-free survival, and Panel B shows the results of the Cox proportional-hazards models for the comparisons of disease-free survival, freedom from recurrence of breast cancer, freedom from recurrence of breast cancer at a distant site, and overall survival, according to treatment group, among all the patients and according to chemotherapy cohort. The solid vertical lines at 0.83 , $0.81,0.88$, and 0.74 indicate the overall hazard-ratio estimates for the four end points, respectively. In the analysis of disease-free survival, the hazard ratio is for breast-cancer recurrence, a second invasive cancer, or death. In the analyses of freedom from breast cancer and freedom from recurrence of breast cancer at a distant site, the hazard ratios are for recurrence. In the overall survival analysis, the hazard ratio is for death. The $P$ value for the comparison among all patients was obtained by means of a stratified log-rank test; the P value for the assessment of treatment-effect heterogeneity according to chemotherapy cohort was calculated by means of a test of treatment by chemotherapy cohort interaction from a stratified Cox proportional-hazards model. The $x$ axis is scaled according to the natural logarithm of the hazard ratio. The size of the squares is inversely proportional to the standard error of the hazard ratio. The median follow-up was 67 months.

Recurrence of breast cancer at a distant site was reported in 185 patients (9.1\%), with no significant difference between those assigned to tamoxifen plus ovarian suppression and those assigned to tamoxifen alone (hazard ratio for recurrence, $0.88 ; 95 \% \mathrm{CI}, 0.66$ to $1.18 ; \mathrm{P}=0.40$ ) (Fig. 3B). Death was reported in 106 patients (5.2\%); 4 patients died without a breast-cancer recurrence or a second invasive cancer. Overall survival at 5 years was $96.7 \%$ (95\% CI, 95.2 to 97.7) among patients assigned to tamoxifen plus ovarian suppression and $95.1 \%$ (95\% CI, 93.4 to 96.3) among those assigned to tamoxifen alone (hazard ratio for death, $0.74 ; 95 \% \mathrm{CI}, 0.51$ to 1.09; $\mathrm{P}=0.13$ ) (Fig. 2B).

Among patients who did not receive chemotherapy, more than $95 \%$ remained free from breast cancer at 5 years in each group (Fig. 3C), with few distant recurrences (Fig. 3D), and $32.9 \%$ of first events (a second invasive cancer or death) were not related to breast cancer (Table S3 in the Supplementary Appendix). Most recurrences of breast cancer were in patients who remained premenopausal after receiving chemo- 


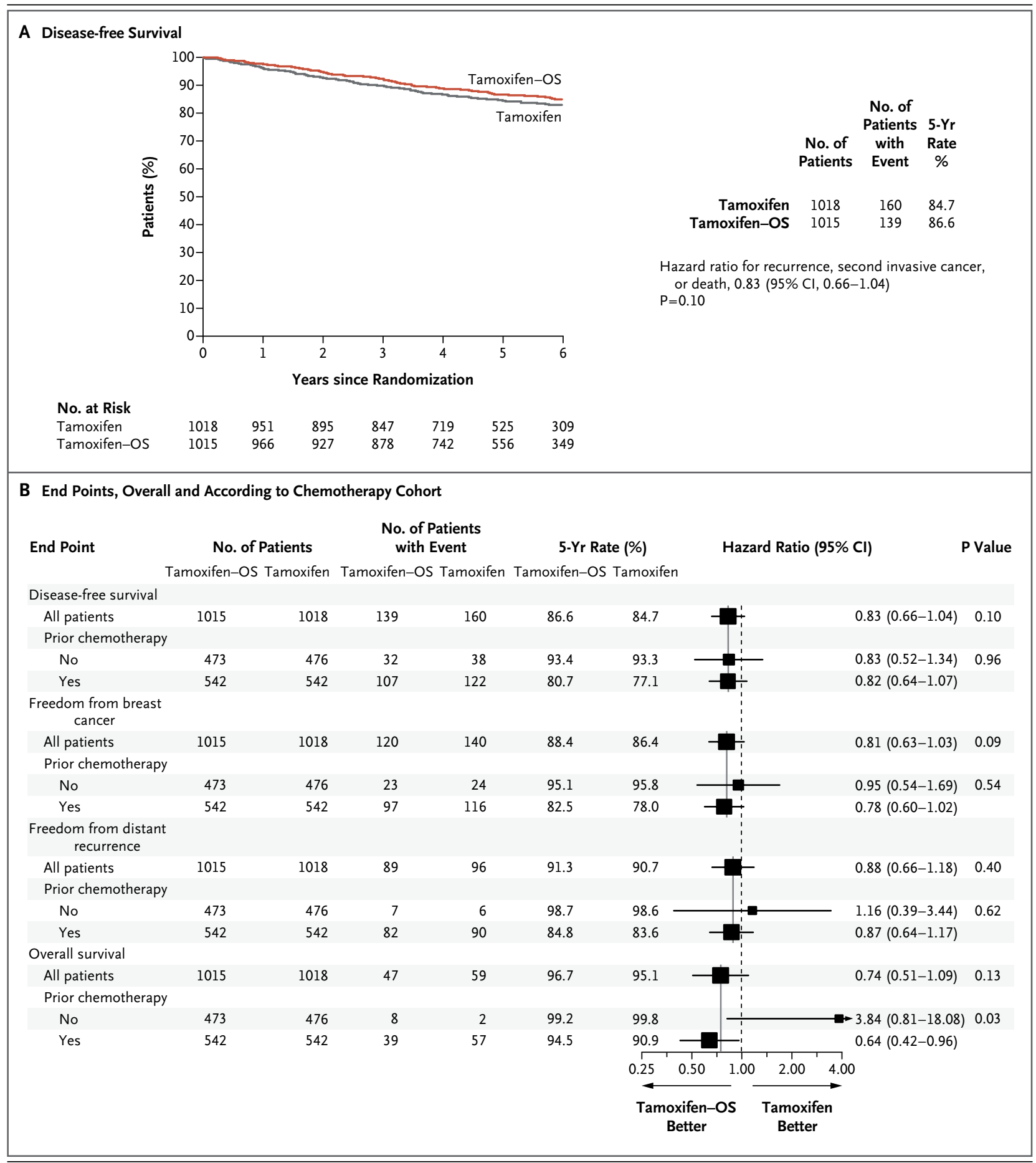

therapy (Fig. 3E). In this cohort, the rate of $78.0 \%$ (95\% CI, 74.0 to 81.5 ) among those asfreedom from breast cancer at 5 years was $82.5 \%$ signed to receive tamoxifen alone (hazard ratio (95\% CI, 78.8 to 85.6 ) among those assigned to for recurrence, 0.78 ; $95 \%$ CI, 0.60 to 1.02 ). In the receive tamoxifen plus ovarian suppression and chemotherapy cohort, among patients assigned 
to exemestane plus ovarian suppression, $85.7 \%$ ( $95 \%$ CI, 82.3 to 88.5 ) remained free from breast cancer at 5 years (Fig. 3E).

Most recurrences of breast cancer at a distant site occurred in the patients who had received chemotherapy previously. The rates of freedom from distant recurrence at 5 years in this cohort were as follows: $83.6 \%$ among patients assigned to tamoxifen alone, $84.8 \%$ among those assigned to tamoxifen plus ovarian suppression, and $87.8 \%$ among those assigned to exemestane plus ovarian suppression (Fig. 3F).

More than $90 \%$ of the deaths occurred in patients who had received chemotherapy previously. Overall survival at 5 years in the chemotherapy cohort was $94.5 \%$ (95\% CI, 92.0 to 96.2 ) among patients assigned to tamoxifen plus ovarian suppression, as compared with $90.9 \%$ (95\% CI, 87.9 to 93.2) among those assigned to tamoxifen alone (hazard ratio for death, 0.64; 95\% CI, 0.42 to 0.96) (Fig. 2B, and Fig. S5 in the Supplementary Appendix).

A total of 350 women younger than 35 years of age participated in the trial, 233 of whom were included in the primary analysis. Among these women, the rate of freedom from breast cancer at 5 years was $67.7 \%$ (95\% CI, 57.3 to 76.0) for patients assigned to tamoxifen alone, $78.9 \%$ (95\% CI, 69.8 to 85.5) for those assigned to tamoxifen plus ovarian suppression, and $83.4 \%$ (95\% CI, 74.9 to 89.3 ) for those assigned to exemestane plus ovarian suppression. In this very young subgroup, $94.0 \%$ of the patients had received chemotherapy previously.

\section{TREATMENT AND ADVERSE EVENTS}

At a median follow-up of 67 months, $25.8 \%$ of the patients were continuing to receive some or all of the protocol-assigned treatment. Tamoxifen was discontinued early, with or without the substitution of alternative endocrine therapy, in $16.7 \%$ of the tamoxifen-ovarian suppression group and $21.7 \%$ of the tamoxifen group (Table S5 in the Supplementary Appendix). Rates of nonadherence with ovarian suppression were $5.0 \%, 9.2 \%, 14.9 \%, 18.3 \%$, and $21.9 \%$ at $0.5,1,2$, 3 , and 4 years after randomization, respectively. Among patients assigned to ovarian suppression, it was achieved entirely through administration of the GnRH agonist triptorelin in $80.7 \%$ of the patients.

Targeted adverse events of grade 3 or higher
Figure 3 (facing page). Kaplan-Meier Estimates of Freedom from Recurrence of Breast Cancer and Freedom from the Recurrence of Breast Cancer at a Distant Site after a Median Follow-up of 67 Months, According to Treatment Assignment.

The estimates for the primary analysis population and the exemestane-ovarian suppression group are summarized for all patients (Panels A and B) and according to chemotherapy cohort (Panels $C$ through F). The 5-year values are based on Kaplan-Meier estimates of the time to an event. In the analyses of freedom from recurrence of breast cancer and freedom from recurrence of breast cancer at a distant site, the hazard ratios are for recurrence of breast cancer and recurrence of breast cancer at a distant site, respectively.

were reported in $31.3 \%$ of the patients assigned to receive tamoxifen plus ovarian suppression, as compared with $23.7 \%$ of those assigned to receive tamoxifen alone (Table 2, and Table S6 in the Supplementary Appendix). Hot flushes, sweating, decreased libido, vaginal dryness, insomnia, depression, musculoskeletal symptoms, hypertension, and glucose intolerance (diabetes) were reported more frequently in the tamoxifen-ovarian suppression group than in the tamoxifen group. Osteoporosis as defined by a T score of less than -2.5 was reported in $5.8 \%$ of the patients assigned to tamoxifen plus ovarian suppression and in $3.5 \%$ of those assigned to tamoxifen alone.

\section{DISCUSSION}

The results of SOFT show that, considering the entire population of patients who underwent randomization, the addition of ovarian suppression to adjuvant tamoxifen did not significantly improve disease-free survival. However, SOFT investigated ovarian suppression in two distinct patient cohorts. The first cohort included 949 premenopausal women for whom adjuvant tamoxifen without chemotherapy was considered to be suitable treatment. These patients were predominantly older than 40 years of age, had small, node-negative tumors of low to intermediate grade, and had excellent outcomes with tamoxifen alone after a median follow-up of 67 months. The findings in this cohort do not currently inform us about the clinical relevance of ovarian suppression because one third of the first events were not related to breast cancer, freedom from recurrence exceeded $95 \%$ at 5 years, and addi- 


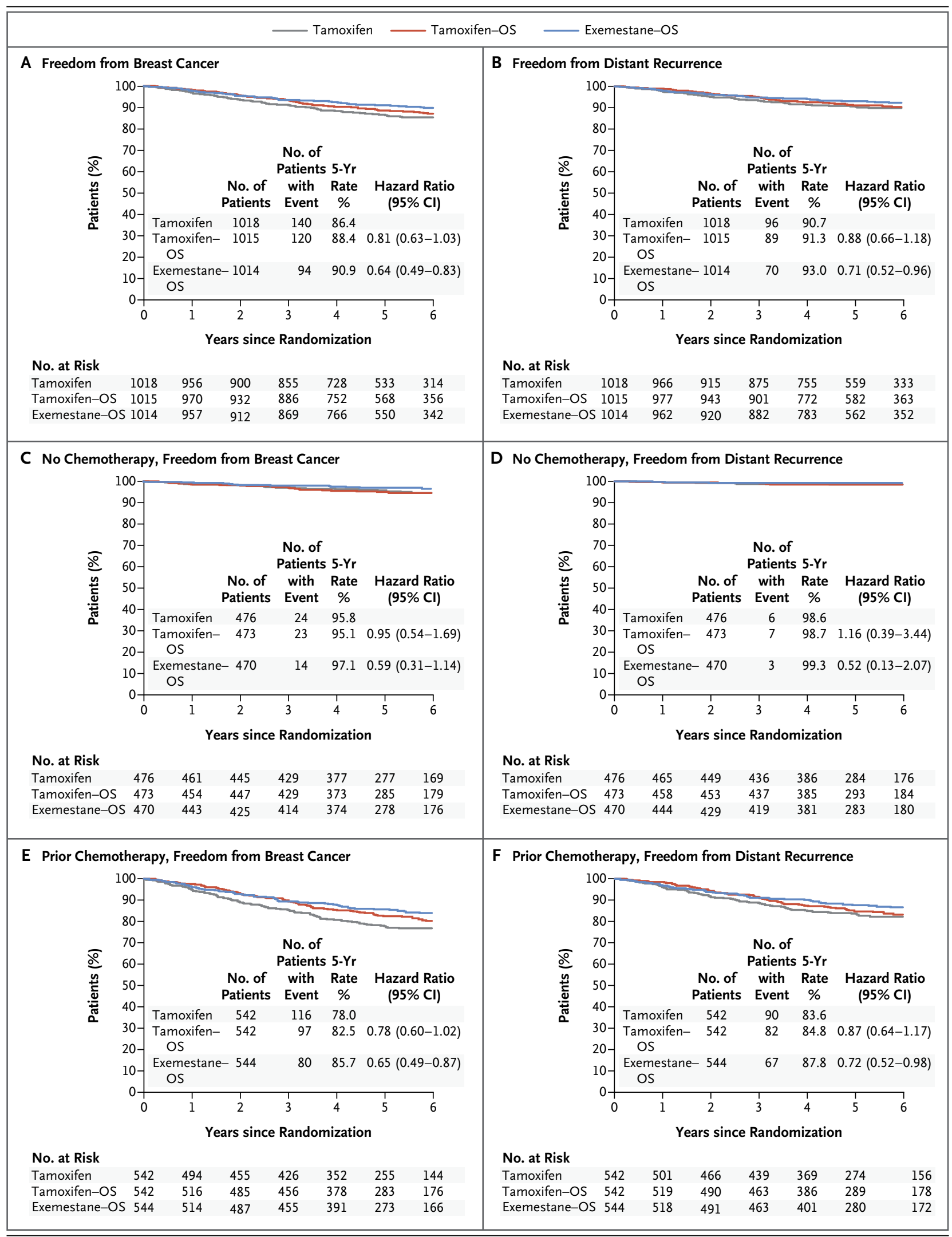

The New England Journal of Medicine 


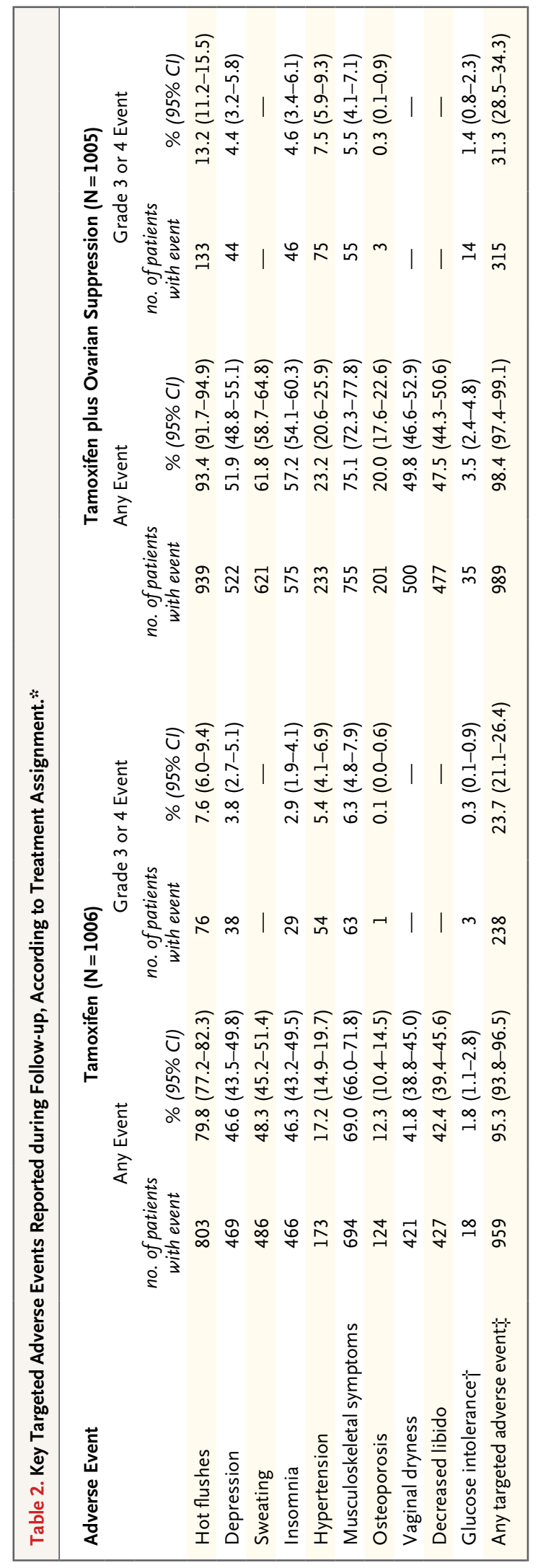

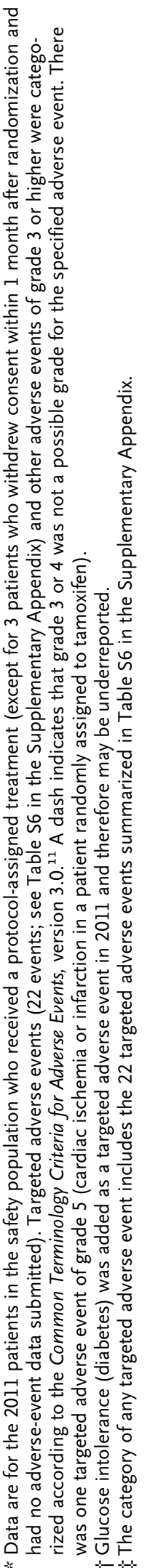

tional recurrences are anticipated with further follow-up.

By contrast, the second cohort included 1084 women who remained premenopausal after completing chemotherapy, as shown by estradiol measurement regardless of menses. The clinicopathological features that warranted prior chemotherapy use $\mathrm{e}^{12}$ and the younger age of the women who remained premenopausal after chemotherapy (median age, 40 years) contributed to the higher risk of recurrence in this cohort than in the cohort that had not received chemotherapy. With a median of 67 months of follow-up, the number of breast-cancer recurrences observed was large enough to indicate that including ovarian suppression as a component of adjuvant therapy can meaningfully reduce recurrences in this cohort (Fig. 3E).

For women with ovarian estrogen production after chemotherapy, we had hypothesized that therapeutic ovarian suppression would provide a benefit similar to that observed with chemotherapy-induced amenorrhea. ${ }^{5-7}$ Resilience of ovarian function to chemotherapy correlates with younger age. Chemotherapy-induced ovarian suppression is common in older premenopausal women and is associated with improved breast-cancer outcomes..$^{5-7}$ The SOFT design for the chemotherapy cohort targeted younger patients by mandating that only women who remained premenopausal or reverted to premenopausal status could be enrolled. Previous trials evaluating ovarian suppression were confounded by the inclusion of women who became permanently postmenopausal from chemotherapy or who had an unknown or negative hormone-receptor status; these trials also lacked a 5-year tamoxifen control group. ${ }^{13-15}$

When SOFT was planned, it was hypothesized that tamoxifen plus ovarian suppression would reduce the relative risk of breast-cancer recurrence, a second invasive cancer, or death by $25 \%$, as compared with tamoxifen alone, and furthermore, that exemestane plus ovarian suppression would reduce the relative risk by $25 \%$, as compared with tamoxifen plus ovarian suppression. After adjustment for covariates in the multivariable Cox model, tamoxifen plus ovarian suppression resulted in a $22 \%$ reduction in the relative risk of breast-cancer recurrence, a second invasive cancer, or death $(\mathrm{P}=0.03)$ and a $25 \%$ reduction in the relative risk of breast-cancer recur- 
rence, as compared with tamoxifen alone. After a protocol amendment, the comparison of exemestane plus ovarian suppression with tamoxifen plus ovarian suppression, on the basis of a combined analysis with TEXT, showed a $28 \%$ reduction in the relative risk of breast-cancer recurrence, a second invasive cancer, or death and a 34\% reduction in the relative risk of breastcancer recurrence in the exemestane-ovarian suppression group $(\mathrm{P}<0.001) .^{10}$

Overall, tamoxifen plus ovarian suppression resulted in an absolute improvement of 2.0 percentage points, as compared with tamoxifen alone, in the proportion of patients without recurrent breast cancer at 5 years. In the higherrisk cohort of patients who remained premenopausal after chemotherapy, tamoxifen plus ovarian suppression resulted in an absolute improvement of 4.5 percentage points, as compared with tamoxifen alone, in the proportion of patients without recurrent breast cancer at 5 years; with exemestane plus ovarian suppression, the absolute improvement was 7.7 percentage points, as compared with tamoxifen alone. These observed relative and absolute benefits at 5 years from ovarian suppression plus tamoxifen or ovarian suppression plus exemestane, as compared with tamoxifen alone, compare favorably with the practice-changing results of randomized trials of adjuvant aromatase inhibitors versus tamoxifen in postmenopausal women. ${ }^{16}$

Patients who receive a diagnosis of hormonereceptor-positive breast cancer when they are younger than 35 years of age are a subgroup considered to be at higher risk for adverse outcomes than are older premenopausal women, on the basis of retrospective analyses of data from IBCSG and U.S. Intergroup trials. ${ }^{17,18}$ The results observed in this subgroup in SOFT add to the evidence that ovarian suppression plays an important role in younger premenopausal patients. ${ }^{13-15}$ Among the women younger than 35 years of age, breast cancer recurred within 5 years in approximately one third of the patients assigned to receive tamoxifen alone but in one sixth of those assigned to receive exemestane plus ovarian suppression.

Any benefit from ovarian suppression must be weighed against the adverse effects. Adding ovarian suppression to tamoxifen resulted in increased adverse events - most notably, menopausal symptoms, depression, and adverse events with possible long-term health implications such as hypertension, diabetes, and osteoporosis. When exemestane is combined with ovarian suppression, adverse sexual, musculoskeletal, and bone-density effects are more frequent than with tamoxifen plus ovarian suppression. ${ }^{10}$

Longer follow-up is required, because SOFT is currently underpowered, and the overall survival analysis is premature after $5 \%$ of patients have died. The combined analysis from TEXT and SOFT showed that adjuvant endocrine therapy with ovarian suppression plus exemestane is significantly more effective than ovarian suppression plus tamoxifen. ${ }^{10}$ The current analysis indicates that in a cohort selected for chemotherapy and persistent premenopausal status, ovarian suppression plus tamoxifen improves outcomes, as compared with tamoxifen alone, with the most striking differences observed among younger patients.

We conclude that adding ovarian suppression to tamoxifen did not provide a significant benefit in the overall population of premenopausal women in this trial. However, in the cohort of women who had a sufficient risk of recurrence to warrant adjuvant chemotherapy and who had premenopausal estradiol levels despite chemotherapy, ovarian suppression in addition to tamoxifen reduced the risk of breast-cancer recurrence, as compared with tamoxifen alone. Ovarian suppression combined with an aromatase inhibitor further reduced the risk of recurrence, as compared with tamoxifen-based therapy, in this higher-risk premenopausal cohort.

Supported by Pfizer, Ipsen, the International Breast Cancer Study Group, and the National Cancer Institute.

Disclosure forms provided by the authors are available with the full text of this article at NEJM.org.

The authors' affiliations are as follows: the Peter MacCallum Cancer Centre, St Vincent's Hospital, University of Melbourne, Melbourne, VIC (P.A.F.), the Australia and New Zealand Breast Cancer Trials Group, University of Newcastle, Newcastle, NSW (P.A.F., A.S.C.), and the University of Sydney, Sydney (A.S.C.) - all in Australia; the International Breast Cancer Study Group (IBCSG) Statistical Center, Dana-Farber Cancer Institute (M.M.R., A.G.-H., R.D.G.), Harvard Medical School (M.M.R., R.D.G.), Susan F. Smith Center for Women's Cancers, Dana-Farber Cancer Institute, Harvard Medical School and Alliance for Clinical Trials in Oncology (H.J.B., E.P.W.), IBCSG Statistical Center, Frontier Science and Technology Research Foundation (K.N.P.), and Harvard School of Public Health, Frontier 
Science and Technology Research Foundation (R.D.G.) - all in Boston; University of Chicago Medical Center and Alliance for Clinical Trials in Oncology, Chicago (G.F.F.); National Institute of Oncology and International Breast Cancer Study Group, Budapest, Hungary (I.L.); University Hospital 12 de Octubre, Madrid (E.C.), Vall d'Hebron Institute of Oncology and Vall d'Hebron University Hospital (M.B.), and SOLTI Group (E.C., M.B., M.A.C.), Barcelona, and Instituto Valenciano de Oncologia, Valencia (M.A.C.) - all in Spain; Institut Bergonié Comprehensive Cancer Center, Université de Bordeaux, INSERM Unité 916, Bordeaux (H.R.B.), and Centre Eugène Marquis, Université de Rennes, Rennes (P.K.) - both in France; European Organization for Research and Treatment of Cancer, Brussels (H.R.B., P.K.); Salvatore Maugeri Foundation and IBCSG, Pavia (G.A.D.P.), and the European Institute of Oncology and International Breast Cancer Study Group (M.C., A.G.) and IBCSG Central Pathology Center, European Institute of Oncology, University of Milan, Milan (G.V.) - all in Italy; the Angeles Clinic and Research Institute and SWOG, Santa Monica, CA (S.M.); University of Pittsburgh Cancer Institute, University of Pittsburgh Medical Center CancerCenter, and the ECOG-ACRIN Cancer Research Group, Pittsburgh (N.E.D.); Massey Cancer Center, Virginia Commonwealth University School of Medicine and NRG Oncology, Richmond (C.E.G.); Tom Baker Cancer Centre and National Cancer Institute of Canada Clinical Trials Group, Calgary, AB, Canada (B.A.W.); Weston Park Hospital, Sheffield, and National Cancer Research Institute Breast Cancer Clinical Studies Group and Institute of Cancer Research Clinical Trials and Statistics Unit, London — all in the United Kingdom (R.C.); German Breast Group and the Department of Obstetrics and Gynecology, University Medical Center Regensburg, Regensburg, Germany (S.B.); Mayo Clinic and Alliance for Clinical Trials in Oncology, Rochester, MN (J.N.I.); and University Hospital Inselspital (M.R.-P.) and IBCSG Coordinating Center (M.R.-P., R.M., B.R.), Bern, Switzerland.

REFERENCES

1. Eifel P, Axelson JA, Costa J, et al. National Institutes of Health Consensus Development Conference Statement: adjuvant therapy for breast cancer, November 1-3, 2000. J Natl Cancer Inst 2001;93:97989.

2. Goldhirsch A, Glick JH, Gelber RD, Coates AS, Senn HJ. Meeting highlights: International Consensus Panel on the Treatment of Primary Breast Cancer. J Clin Oncol 2001;19:3817-27.

3. LHRH-Agonists in Early Breast Cancer Overview Group. Use of luteinisinghormone-releasing hormone agonists as adjuvant treatment in premenopausal patients with hormone-receptor-positive breast cancer: a meta-analysis of individual patient data from randomised adjuvant trials. Lancet 2007;369:1711-23.

4. Griggs JJ, Somerfield MR, Anderson $\mathrm{H}$, et al. American Society of Clinical Oncology endorsement of the Cancer Care Ontario practice guideline on adjuvant ovarian ablation in the treatment of premenopausal women with early-stage invasive breast cancer. J Clin Oncol 2011;29: 3939-42. [Erratum, J Clin Oncol 2012;30: 1398.]

5. Pagani O, O'Neill A, Castiglione M, et al. Prognostic impact of amenorrhoea after adjuvant chemotherapy in premenopausal breast cancer patients with axillary node involvement: results of the International Breast Cancer Study Group
(IBCSG) Trial VI. Eur J Cancer 1998;34: 632-40.

6. International Breast Cancer Study Group. Tamoxifen after adjuvant chemotherapy for premenopausal women with lymph node-positive breast cancer: International Breast Cancer Study Group Trial 13-93. J Clin Oncol 2006;24:1332-41.

7. Swain SM, Jeong J-H, Wolmark N. Amenorrhea from breast cancer therapy - not a matter of dose. $\mathrm{N}$ Engl J Med 2010;363:2268-70.

8. Partridge $\mathrm{AH}$, Pagani $\mathrm{O}$, Abulkhair $\mathrm{O}$, et al. First international consensus guidelines for breast cancer in young women (BCY1). Breast 2014;23:209-20.

9. Regan MM, Pagani O, Fleming GF, et al. Adjuvant treatment of premenopausal women with endocrine-responsive early breast cancer: design of the TEXT and SOFT trials. Breast 2013;22:1094-100. 10. Pagani O, Regan MM, Walley BA, et al Adjuvant exemestane with ovarian suppression in premenopausal breast cancer N Engl J Med 2014;371:107-18.

11. Cancer Therapy Evaluation Program. Common terminology criteria for adverse events, version 3.0 (http://ctep.cancer .gov/protocolDevelopment/electronic applications/docs/ctcaev3.pdf)

12. Regan MM, Pagani O, Walley B, et al. Premenopausal endocrine-responsive early breast cancer: who receives chemotherapy? Ann Oncol 2008;19:1231-41.
13. International Breast Cancer Study Group. Adjuvant chemotherapy followed by goserelin versus either modality alone for premenopausal lymph node-negative breast cancer: a randomized trial. J Natl Cancer Inst 2003;95:1833-46.

14. Davidson NE, O'Neill AM, Vukov AM, et al. Chemoendocrine therapy for premenopausal women with axillary lymph node-positive, steroid hormone receptorpositive breast cancer: results from INT 0101 (E5188). J Clin Oncol 2005;23:597382.

15. Hackshaw A, Baum M, Fornander T, et al. Long-term effectiveness of adjuvant goserelin in premenopausal women with early breast cancer. J Natl Cancer Inst 2009;101:341-9.

16. Dowsett $M$, Cuzick J, Ingle J, et al. Meta-analysis of breast cancer outcomes in adjuvant trials of aromatase inhibitors versus tamoxifen. J Clin Oncol 2010;28: 509-18.

17. Aebi S, Gelber S, Castiglione-Gertsch $\mathrm{M}$, et al. Is chemotherapy alone adequate for young women with oestrogen-receptor-positive breast cancer? Lancet 2000; 355:1869-74.

18. Goldhirsch A, Gelber RD, Yothers G, et al. Adjuvant therapy for very young women with breast cancer: need for tailored treatments. J Natl Cancer Inst Monogr 2001;30:44-51.

Copyright (c) 2014 Massachusetts Medical Society.

RECEIVE IMMEDIATE NOTIFICATION WHEN AN ARTICLE

IS PUBLISHED ONLINE FIRST

To be notified by e-mail when Journal articles

are published Online First, sign up at NEJM.org. 